\title{
El impacto de la ultraderecha en Twitter durante las elecciones españolas de 2019
}

\section{The impact of the far-right on Twitter during the 2019 Spanish elections \\ O impacto da extrema direita no Twitter durante as eleições espanholas de 2019}

Frederic Guerrero-Solé, Universitat Pompeu Fabra, Barcelona, España (frederic.guerrero@upf.edu)

Lluís Mas-Manchón, Universitat Pompeu Fabra, Barcelona, España (lluis.mas@upf.edu) Toni Aira, UPF Barcelona School of Management (UPF-BSM), Barcelona, España (toni.aira@bsm.upf.edu)

RESUMEN | Las dos elecciones españolas de 2019, celebradas el 28 de abril (28A) y el 10 de noviembre (10N), estuvieron marcadas por la irrupción del partido Vox, populista de extrema derecha, que pasó de 23 a 52 diputados. El objetivo de esta investigación es analizar el impacto de su irrupción en la estructura de las comunidades (Smith et al., 2014) de las redes de debate político durante estas dos elecciones en Twitter. El estudio compara además las estructuras de las dos elecciones de 2019 con las de 2015 y 2016, así como las distancias políticas entre los principales partidos españoles. Las muestras analizadas se componen de más de 1,5 millones de tuits publicados durante las campañas electorales del $28 \mathrm{~A}$ y el $10 \mathrm{~N}$. Aunque las investigaciones anteriores muestran que, en general, los patrones de interacción y las estructuras de las redes tienden a permanecer estables después de la repetición de unas elecciones, esta investigación muestra un cambio sustancial en la estructura de las redes de intersección de comunidades de retuiteadores RON (Guerrero-Solé, 2017) y en las distancias entre partidos. Específicamente, la investigación evidencia un incremento de la distancia entre los dos principales bloques políticos españoles -el formado por la triple derecha (PP, Cs y Vox) y el constituido por partidos de izquierda y nacionalistasy (2) la ubicación de Vox en el centro del clúster de las opciones de derecha.

PALABRAS CLAVE: Twitter; medios sociales; comunicación política; elecciones; España; ultraderecha española; Vox; estructura de las comunidades. 
ABSTRACT / The two 2019 Spanish elections, held on April 28 (28A) and on November 10 $(10 N)$, were marked by the irruption of the far-right populist party Vox, which went from 23 to 52 deputies. The aim of this research is to analyze the impact of its emergence in the structure of the communities (Smith et al., 2014) of political debate networks on Twitter during these two elections. Thestudy also compares the structures of the two 2019 elections with those of 2015 and 2016, as well as the political distances between the main Spanish parties. The analyzed sample is made up of more than 1.5 million tweets published during the electoral campaigns of $28 \mathrm{~A}$ and $10 \mathrm{~N}$. Although previous research shows that, in general, interaction patterns and networks structures tend to remain stable after the repetition of an election this research shows a substantial change in the structure of $R O N$ communities (Guerrero-Solé, 2017) and in the distances between parties. Specifically, the research shows an increase in the distance between the two main Spanish political blocs -the one formed by the triple right wing (PP, Cs and Vox) and the one constitudes by left and nationalist parties- and (2) the location of Vox in the center of the right-wing cluster.

KEYWORDS: Twitter; social media; political communication; election campaign; Spain; Spanish far-right; Vox; structure of communities.

RESUMO|As duas eleições espanholas de 2019, celebradas no 28 de abril (28A) e o 10 de novembro (10N), foram marcadas pela irrupção do partido populista de extrema direita Vox, que passou de 23 a 52 deputados. O objetivo desta pesquisa é analisar o impacto da irrupção de Vox na estrutura das comunidades (Smith et al., 2014) das redes de debate político durante as duas eleições no Twitter. O estudo compara também as estruturas das duas eleições de 2019 com as de 2015 e 2016, além das distancias políticas entre os principais partidos espanholes. As mostras analisadas se compõem de mais de 1,5 milhões de tuítes publicados durante as campanhas eleitorais do $28 \mathrm{~A}$ e o $10 \mathrm{~N}$. Ainda que as pesquisas anteriores mostremm que, em geral, os padrões de interação e as estruturas das redes tendem a permanecer estáveis depois da repetição de umas eleições, esta pesquisa mostra uma mudança significativa na estrutura de redes de interseção das comunidades RON (GuerreroSolé, 2017) e nas distâncias entre partidos. Em particular, a pesquisa evidencia (1) um incremento das distâncias entre os dos principais blocos políticos espanhóis, e as distâncias entre o bloco da triple direita (PP, Cs e Vox) e o bloco dos partidos de esquerda e nacionalistas, e (2) a localização de Vox no centro do cluster das opções da direita.

PALAVRAS-CHAVE: Twitter; mídia social; comunicação política; eleições; Espanha; extrema dereita espanhola; Vox; estruturas das comunidades. 


\section{INTRODUCCIÓN}

Las redes sociales, como espacios de debate público, son actualmente una fuente privilegiada de acceso al conocimiento sobre las interacciones entre los diferentes actores sociales. En el caso de los debates políticos, Twitter se ha situado como uno de los espacios principales de interacción. Sus características, así como el hecho de que el acceso a los datos mediante las API (Application Programming Interface) y su procesamiento y análisis son mucho más accesibles que el del resto de redes sociales (Bruns, 2019a), la ha convertido en objeto de investigación preferente en el campo de la comunicación política actual. Son muchos los estudios que, desde perspectivas diferentes, han analizado las interacciones entre actores sociales en conversaciones políticas en Twitter. Desde los pioneros trabajos de Tumasjan y sus colegas (2010), cuyo objetivo era predecir resultados electorales con base en la actividad en Twitter, hasta la miríada de estudios en una gran diversidad de contextos, la investigación en comunicación política ha considerado esta red de microblogging como una fuente de datos fundamental. Uno de los campos de investigación que más se ha estudiado en Twitter es la detección de la estructura de comunidades. Los resultados, que en su mayoría concluyen que las interacciones políticas son de carácter homofílico y que las comunidades políticas tienden a un mayor distanciamiento y polarización, han puesto el foco actualmente en las consecuencias derivadas de las llamadas cámaras de resonancia (echo chambers) y las burbujas de filtro (filter bubbles) (Bruns, 2019b).

Otro aspecto que ha llamado la atención de los investigadores es la influencia de los populismos y de los movimientos políticos de extrema derecha en las redes sociales y el rol que juegan en la difusión de sus mensajes (Daniels, 2018). Así, desde la victoria de Donald Trump en las elecciones norteamericanas en 2016, la preocupación por el impacto negativo que pueden tener las redes en las sociedades actuales ha ido en aumento. A esto se ha unido el temor a la propagación de las noticias falsas (fake news), que sitúan de nuevo a las redes sociales en el foco (Allcott \& Gentzkow, 2017).

Este trabajo tiene como objetivo analizar las interacciones entre actores políticos en los debates en Twitter durante las campañas electorales de abril y noviembre de 2019 en España. El estudio se centra, específicamente, en el impacto que el partido populista de ultraderecha Vox tuvo en la estructura de comunidades de las redes en ambas elecciones. Para ello, utilizamos el método RON (Retweet Overlap Network) de identificación de comunidades. Los resultados obtenidos se comparan con los ya publicados sobre las elecciones generales de 2015 y 2016 en España (Guerrero-Solé, 2018; Mas Manchón \& Guerrero-Solé, 2019), en las que Vox era un partido residual. Asimismo, se analizan las distancias políticas a través de los datos obtenidos con el método RON, y se comparan con las de las anteriores elecciones. 
Este trabajo añade conocimiento al impacto de la aparición de la ultraderecha como una fuerza política de relevancia en la dinámica de estructuración de las redes sociales.

\section{Twitter y comunicación política}

No hay duda de que Twitter se ha convertido en una de las redes sociales principales para el debate político y, por sus características y la facilidad de acceso a sus datos, en la red predilecta de los investigadores en comunicación, específicamente en los casos de análisis numéricos con grandes cantidades de datos (Arcila Calderón et al., 2021). Esto se comprende más aún si se consideran las dificultades para obtener datos de redes sociales como Facebook e Instagram después del escándalo de Cambridge Analytica en 2018 y que ha provocado lo que Bruns (2019a) ha bautizado como Apicalypse, o la imposibilidad de los académicos de poder escrutar de forma crítica e independiente a las grandes plataformas.

Entre el amplio abanico de investigaciones centradas en Twitter (ver el trabajo recopilatorio de Jungherr en 2014 sobre la investigación en comunicación política publicada hasta la fecha), destacan aquellas centradas en detectar comunidades o grupos de interacción (Conover et al., 2011; Boutet et al., 2012). Una red social tiene estructura de comunidades cuando puede dividirse en subgrupos de usuarios con conexiones intensas entre ellos (Xie \& Szymanski, 2011). Fortunato (2010) revisa algunos de los algoritmos de detección de comunidades más utilizados. Entre ellos, el LPA (Label Propagation Algorithm) propuesto por Raghavan y sus colegas (2007) fue utilizado por Boutet y colaboradores (2012) en su análisis de las elecciones británicas de 2010. Para Conover et al. (2011), el LPA es un método útil para detectar clústeres políticos, asumiendo que los usuarios retuitean mensajes afines a su propia ideología. La misma premisa es la base del método de análisis RON de redes de intersección de comunidades de retuiteadores (Guerrero-Solé, 2017) que ha sido utilizado para el análisis de las comunidades políticas en España. Azaouzi y sus colegas (2019) recogen el estado de la cuestión de los diferentes métodos de detección de comunidades en redes sociales de gran escala.

En general, las investigaciones centradas en la estructura de comunidades de las redes de debate político concluyen que están sensiblemente polarizadas, siguiendo el patrón de lo que Smith y colaboradores (2014) llaman multitudes polarizadas (polarized crowds). La topología de las multitudes polarizadas se explica, fundamentalmente, a partir de la homofilia (Hanna et al., 2013; Bessi et al., 2016). Los grupos ideológicos tienden al aislamiento y a la ignorancia mutua, con poco diálogo entre partidos y votantes (Marín-Dueñas et al., 2019), dando lugar a lo que se han denominado cámaras de resonancia (Sunstein, 2001, 2009, 2017; Pariser, 2011; Barberá et al., 2015; del Vicario et al., 2016). Este hecho ha generado una gran 
preocupación acerca de cómo las redes sociales están socavando la democracia (Tucker et al., 2017). Sin embargo, hay otras investigaciones que ponen en duda el impacto de las redes en la polarización política y muestran que la exposición a diferentes versiones de la realidad es hoy en día más frecuente debido a multitud de factores contextuales (Wojcieszak \& Mutz, 2009; Bakshy et al., 2015; Boxell et al., 2017). Estas investigaciones ponen en duda la existencia misma de las cámaras de resonancia (Bruns, 2019b).

\section{Populismo y extrema derecha}

A pesar de las dudas, la cuestión acerca de la polarización continúa ocupando un lugar de relevancia dentro del conjunto de preocupaciones de las democracias actuales. A ello se han unido dos factores más que han pasado a ser objeto preferente de las investigaciones recientes: la difusión de rumores y noticias falsas, y el auge del populismo (Mudde, 2004; Rooduijn, 2014), sobre todo el de extrema derecha, en las democracias occidentales. El crecimiento de la extrema derecha populista es causa de preocupación en Europa (Golder, 2016). En la actualidad, partidos de extrema derecha tienen un rol de relevancia en países como Hungría, Francia o Polonia, y han participado en gobiernos de coalición en Austria, Italia u Holanda (Golder, 2016). Esta tendencia al crecimiento de la extrema derecha se considera como una amenaza al liberalismo (Ikenberry, 2018) y representa la culminación de lo que Von Beyme califica como la tercera ola de extremismo de derechas. Las características de la extrema derecha son el etnocentrismo, el anti-pluralismo, antindividualismo, un nacionalismo hipertrofiado y actitudes misionarias (von Beyme, 1988), así como su apuesta por las acciones radicales, que incluyen la violencia (Norris, 2005; Crosset et al., 2019).

El populismo ha convertido a las redes sociales en el principal instrumento de difusión de sus mensajes simplistas y emocionales (Taggart, 2000; Klinger \& Svensson, 2015), que tienden a reducir la complejidad de la realidad (Engesser et al., 2017a). Aunque la literatura sobre populismo es amplia y tiene una larga tradición, la victoria de Donald Trump en las elecciones norteamericanas de 2016 y el auge de los movimientos de ultraderecha en las democracias occidentales Hungría, Italia, Austria o el Reino Unido, en Europa, y Brasil en Sudamérica son ejemplos evidentes-, ha impulsado la investigación sobre la contribución de las redes sociales al éxito del populismo. Este siempre se ha servido de los medios alternativos a los tradicionales para difundir su mensaje (Engesser et al., 2017b). Hay autores que han relacionado el movimiento populista con las nuevas tecnologías, e incluso hay posiciones que explican el populismo a partir de la evolución de las tecnologías de la comunicación. De esta manera, las redes sociales son un elemento fundamental para la existencia y el éxito del populismo. La desintermediación favorecida por las redes sociales (Engesser et al., 2017a; Bracciale \& Martella, 2017) 
ha provocado que los partidos populistas gocen de mayor libertad en la crítica a las élites (Klinger \& Svensson, 2015; Suiter et al., 2018).

La ultraderecha ha hecho un uso estratégico de las redes sociales. Como apuntan Crosset y sus colegas (2019), una gran mayoría de las investigaciones sobre extrema derecha y medios digitales se ha centrado en el análisis de contenido (Atton, 2006; Berger, 2018; McSwiney, 2020), la difusión de desinformación en los medios de extrema derecha (Faris et al., 2017; Pierri et al., 2020), la influencia de los líderes de ultraderecha y sus medios (Pérez Curiel, 2020), o la transnacionalidad del discurso de extrema derecha (Caiani \& Kröll, 2015; Froio \& Ganesh, 2019). Sin embargo, también ha habido un interés creciente por analizar las estructuras de los partidos de ultraderecha en las redes sociales (Caiani \& Wagemann, 2009; Caiani \& Parenti, 2013). En este sentido, Twitter ofrece unas características exclusivas para el análisis de las interacciones en línea de los usuarios de extrema derecha (Crosset et al., 2019). McSwiney (2020), por ejemplo, ha analizado la estructura en comunidades y la actividad en Twitter (y también en Facebook) de la ultraderecha australiana, concluyendo que no forman una estructura homogénea y que muchos de los partidos de extrema derecha son una radicalización de posiciones de centroderecha.

En España, Caiani y Parenti (2013) analizaron la estructura en línea de los medios de extrema derecha en Internet. Una de las conclusiones era la escasa utilización de los medios digitales por parte de la extrema derecha española. Si los utilizaban, lo hacían para difundir contenido nostálgico del fascismo, nazismo y franquismo, sin una estrategia transnacional. Estudios más recientes se han centrado en la influencia en Twitter de la extrema derecha española en campaña, como en las elecciones autonómicas andaluzas de 2018 (Rivas-de-Roca et al., 2020).

\section{Vox, la ultraderecha en España}

El bipartidismo había caracterizado desde principios de los años 1980 a la política española. Los gobiernos del PSOE y PP se habían alternado hasta las elecciones de la década de 2010. Sin embargo, las crisis económica e institucional transformaron el escenario político y dos nuevas fuerzas aparecieron en él. La izquierda de Podemos y el conglomerado de centroderecha de Ciudadanos emergieron como alternativas a los partidos tradicionales, a la vez que los llamados partidos nacionalistas y separatistas de Cataluña y el País Vasco reforzaron sus posiciones en el Congreso de los Diputados (ver tabla 1).

Sin embargo, en 2019 Vox consiguió convertirse en la tercera fuerza política por representación parlamentaria, pasando de 24 diputados en las elecciones del 28 de abril de 2019 a 52 en las del 10 de noviembre del mismo año (Minder, 2019). Hasta las elecciones de abril de 2019, Vox no había conseguido ni un solo escaño. Su primer gran éxito electoral fue en las elecciones autonómicas andaluzas, en las 
Partido

\begin{tabular}{ccc}
\hline PSOE & 123 & 120 \\
\hline PP & 66 & 89 \\
\hline VOX & 24 & 52 \\
\hline UP & 42 & 35 \\
\hline ERC & 15 & 13 \\
\hline CS & 57 & 10 \\
\hline JxCAT & 7 & 8 \\
\hline PNV & 6 & 6 \\
\hline EH-B & 4 & 5 \\
\hline MP & - & 3 \\
\hline CUP & - & 2 \\
\hline Other & 6 & 7 \\
\hline
\end{tabular}

Tabla 1. Resultados de las dos últimas elecciones generales en 2019 en España

Fuente: Elaboración propia con base en información del Ministerio del Interior de España (http://www.interior.gob.es/elecciones-generales-10n).

que obtuvo $11 \%$ de los votos, lo que supuso 12 diputados del parlamento andaluz. No obstante, las circunstancias políticas en España, el auge del populismo de ultraderecha en otros países europeos y, específicamente, el desafío separatista catalán y la situación derivada del referéndum para la independencia de Cataluña celebrado el 1 de octubre de 2017 (Minder, 2019; Turnbull-Dugarte, 2019; Hedgecoe, 2019), impulsaron al partido de Abascal en 2019, después de haber conseguido un exiguo 0,2\% de los votos en las elecciones de 2015 y 2016. Según Vampa (2020), el elemento territorial es clave para entender el éxito de Vox en 2019. En este sentido, Vox consigue sus mejores resultados en provincias de territorios no movilizados en cuanto a las demandas territoriales. Además, el porcentaje de votos es mayor en aquellas zonas en las que ha habido un mayor influjo de inmigración (Vampa, 2020). Sin embargo, Rinken (2019) pone en duda que las actitudes antiinmigración expliquen el comportamiento de los votantes de Vox.

Así pues, si desde principios de la década de 2010 se observó en España el crecimiento de fuerzas políticas alternativas al bipartidismo - Ciudadanos, de centroderecha, y Podemos, populismo inclusivo de izquierdas (Vampa, 2020) -, en 2019 fue el populismo de ultraderecha el que se situó en un lugar de privilegio dentro del congreso de los diputados. Su éxito puso en alarma tanto a los partidos nacionalistas y de izquierda, por el posible pacto entre la triple derecha (PP, Cs y Vox), como a los de derecha (PP y Cs), que temían la fragmentación del voto de derechas, lo que les obligó a endurecer su discurso (Jones, 2019) y acercarse al de Vox. 
Vox es un partido fundado en 2013 fruto de una escisión del Partido Popular (PP) y como consecuencia de la moderación del PP bajo el liderazgo de Mariano Rajoy (Jones, 2019). Vox había sido un partido irrelevante hasta 2018 (Vampa, 2020). Su actual presidente, Santiago Abascal, había militado en el PP vasco, y fue el artífice de la actual radicalización del partido (Ferreira, 2019). Sus equivalentes políticos a nivel europeo son Marine Le Pen o Geert Wilders, y tienen a Steve Bannon, estratega de Donald Trump, como referente (Ferreira, 2019). Ferreira (2019) clasifica a Vox como partido de ultraderecha, o derecha radical, mediante el análisis de siete características: nacionalismo, nativismo, autoritarismo, antidemocracia, populismo, valores tradicionales y neoliberalismo. Para el autor, el discurso de Vox es más nacionalista que populista.

Vox se define como la voz de la España viva, la garantía de la indisolubilidad de la nación. En su Manifiesto Fundacional, el partido denuncia la degradación del Estado y la corrupción del sistema político que pone al gobierno de la nación a merced de sus enemigos. Siguiendo las pautas de los movimientos populistas, Vox considera que España sufre una crisis sistémica que afecta a su economía y a sus instituciones, así como a su unidad y a su moral colectiva. Esta crisis requiere una actuación que restaure el orden y que haga frente al poder ejercido por la élite política y mediática. Vox es, finalmente, un defensor de la monarquía española y un partido cuya ideología se basa en la nostalgia de un liderazgo fuerte.

\section{Vox y redes sociales}

A principios de la década de 2010, los partidos de ultraderecha españoles apenas utilizaban los medios digitales para difundir sus mensajes, que eran, principalmente, nostálgicos (Caiani \& Parenti, 2013). Sin embargo, a finales de la misma década, Vox, junto con Podemos, ambos partidos españoles de reciente creación, son los que mayor repercusión tienen en redes como Instagram o Twitter (Abuín-Vences \& García-Rosales, 2020). Vox es ahora mismo uno de los partidos más activos en España (Jones, 2019). En general, ha llevado a cabo estrategias de base para expandir su influencia más allá de la intermediación de los medios tradicionales (Turnbull-Dugarte, 2019) y para forzar a sus oponentes políticos a responder a sus provocaciones (Jones, 2019). Además, sus estrategias en Twitter, así como en otras redes sociales como Facebook, Instagram o Tik Tok se caracterizan por la simplicidad, el lenguaje directo y beligerante, y con intención de descalificar al oponente político (Castro Martínez \& Días Morilla, 2021).

Sin embargo, la presencia de la extrema derecha española en las redes sociales ha sido hasta hoy poco investigada. Esta investigación tiene como objetivo ampliar el conocimiento sobre el impacto de Vox en Twitter durante las dos últimas campañas de las elecciones generales de 2019 en España. 
Considerando lo anterior, las preguntas de investigación de este estudio son las siguientes:

PI1. ¿Cómo se estructuraron las comunidades políticas en los debates electorales de abril y noviembre de 2019 y qué diferencias se observan entre las estructuras de 2019 y las de las elecciones de 2015 y 2016 ?

PI1.1. ¿Qué rol juega el populismo de ultraderecha de Vox en estas estructuras?

PI2. ¿Cuáles son las distancias ideológicas entre partidos en las elecciones de abril y noviembre de 2019 y qué diferencias se observan entre estas distancias y las de las elecciones de 2015 y 2016 ?

PI2.1. ¿Cuáles son las distancias de Vox con el resto de los partidos políticos y cómo se interpretan?

\section{MÉTODO Y MUESTRA}

Para responder a las preguntas de investigación utilizaremos el método desarrollado por Guerrero-Solé y López González (2019) llamado retweet overlap network (RON). El método analiza los retuits de los usuarios con mayor difusión en una determinada conversación, y calcula las intersecciones entre las comunidades de retuiteadores de estos usuarios. Esta técnica es análoga a la de cocitación, y permite descubrir la estructura de comunidades, así como de algunos de los atributos latentes de los usuarios que participan en la discusión (Guerrero-Solé, 2018). Además de ser un método eficaz para la identificación de comunidades, también ha sido utilizado para calcular las distancias entre partidos políticos que se han relacionado con la distancia ideológica y con las preferencias de los votantes para formar pactos después de las elecciones (Guerrero-Solé et al., 2014; GuerreroSolé, 2018; Guerrero-Solé \& López-González, 2019). Para una explicación en detalle del método, que utiliza el coeficiente de Jaccard para el cálculo de distancias, veáse Guerrero-Solé y López-González (2019).

Las muestras que se detallan a continuación fueron recogidas mediante la API Search de Twitter (Gómez-Domínguez et al., 2016). Los datos utilizados para el análisis son dos muestras de las campañas electorales del 28 de abril (28A) y del 10 de noviembre $(10 \mathrm{~N})$ de 2019:

EG28A: muestra de 1.259 .324 tuits y retuits publicados durante la campaña electoral en abril de 2019 que contenían la etiqueta (hashtag) \#28A.

EG10N: muestra de 5.081.222 tuits y retuits publicados durante la campaña electoral en noviembre de 2019 con la etiqueta (hashtag) \#10N. 
Los datos fueron almacenados en bases de datos para su proceso y análisis. Como en estudios anteriores, seleccionamos los 1000 usuarios más retuiteados de ambas conversaciones, e identificamos sus comunidades de retuiteadores a partir de los retuits obtenidos durante el proceso de recolección. Posteriormente, utilizando el coeficiente de Jaccard, calculamos numéricamente la intersección entre comunidades. Este valor se utiliza para la construcción de las redes resultantes. De esta manera, dos usuarios están conectados en la red RON si comparten al menos un retuiteador en sus respectivas comunidades de retuiteadores. El hecho de que el coeficiente de Jaccard nos dé un valor que puede ser identificado como la intensidad de la conexión entre usuarios permite también la construcción de las redes resultantes a partir de valores umbral. De esta forma, se identifican las comunidades que están más fuertemente conectadas a través de sus comunidades de retuiteadores, y facilitan la identificación de los atributos latentes del resto de los usuarios de la red.

Posteriormente, identificamos a los usuarios más influyentes en cuatro categorías: partidos y políticos, medios de comunicación, periodistas, y una última categoría en la que se incluye el resto de los usuarios que no pertenecen a ninguna de las tres categorías anteriores.

\section{RESULTADOS}

Para responder a la primera pregunta de investigación sobre la estructura de las comunidades políticas en los debates electorales de abril y noviembre de 2019 y las diferencias observadas entre las estructuras de 2019 y las de las elecciones de 2015 y 2016, construimos las dos redes RON con un valor umbral de 0,01 (figuras 1 y 2).

En estas dos figuras podemos ver la distribución incipiente en comunidades claramente identificables. En ambas, vemos cómo los partidos de derecha (Vox, PP y Cs), se sitúan en un mismo clúster, del mismo modo que hacen los de izquierda (PSOE y UP) junto con los nacionalistas e independentistas catalanes (ERC y JuntsxCAT)

Sin embargo, para tener una imagen más clara de esta estructura, escogimos diferentes valores umbrales, intentando equilibrar el aumento de este valor umbral con la posible pérdida de nodos en la red. De este modo, y como en investigaciones anteriores, escogimos el valor umbral de 0,05 (figura 3).

Como podemos observar en las figuras 3 y 4 , la red se estructuró en comunidades fácilmente identificables, con los clústers de derecha e izquierda más nacionalistas aún más claramente identificados, y con una práctica ausencia de enlaces entre ambos para un umbral de 0,05. 


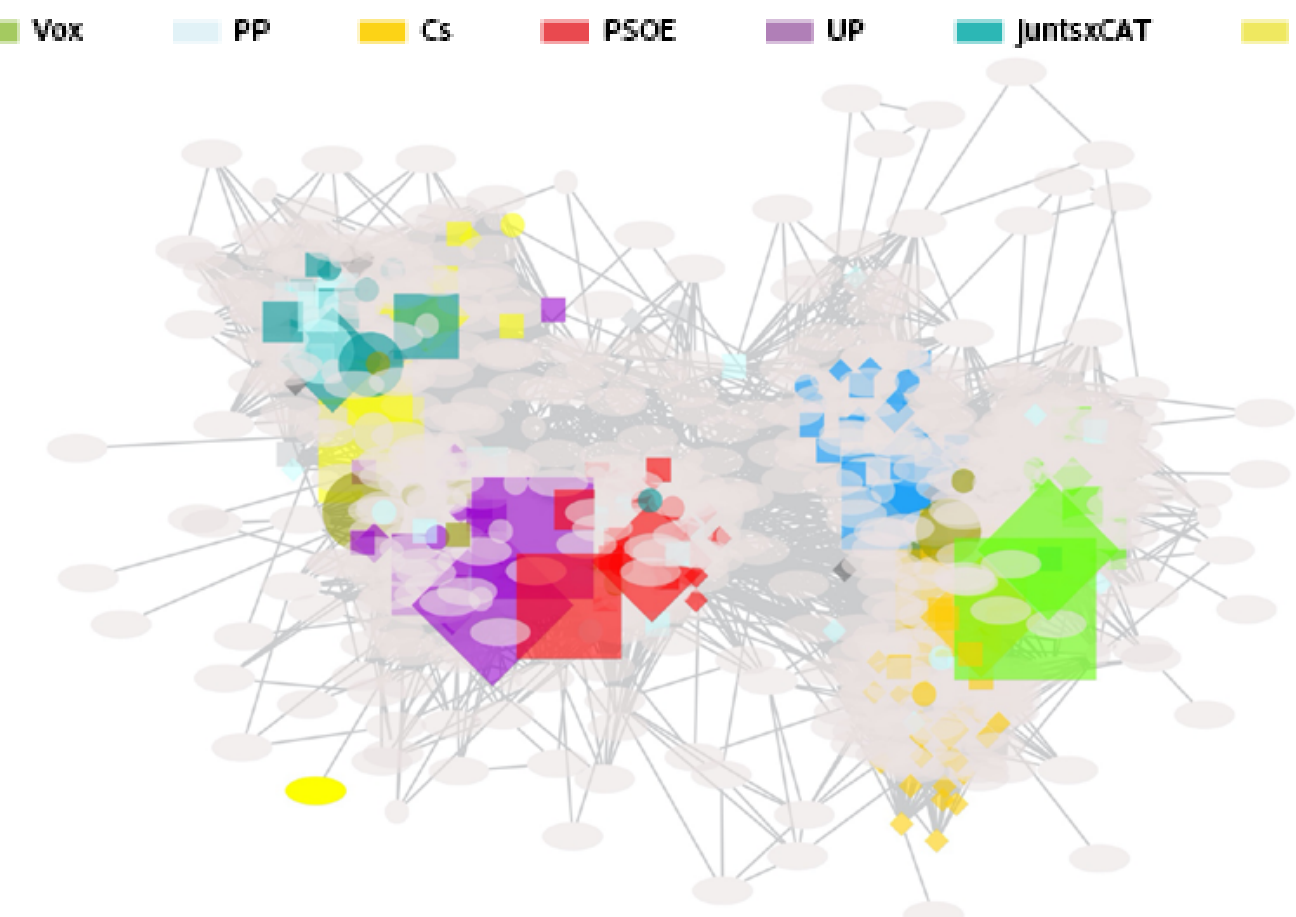

Figura 1. Estructura de comunidades de la red RON para las elecciones del 28 de Abril (28A) de 2019, con valor umbral de 0,01 (Vox-verde claro, PP-azul claro, Cs-naranja, PSOE-rojo, UP-violeta, JuntsxCAT-turquesa, ERC-amarillo).

Fuente: Elaboración propia.

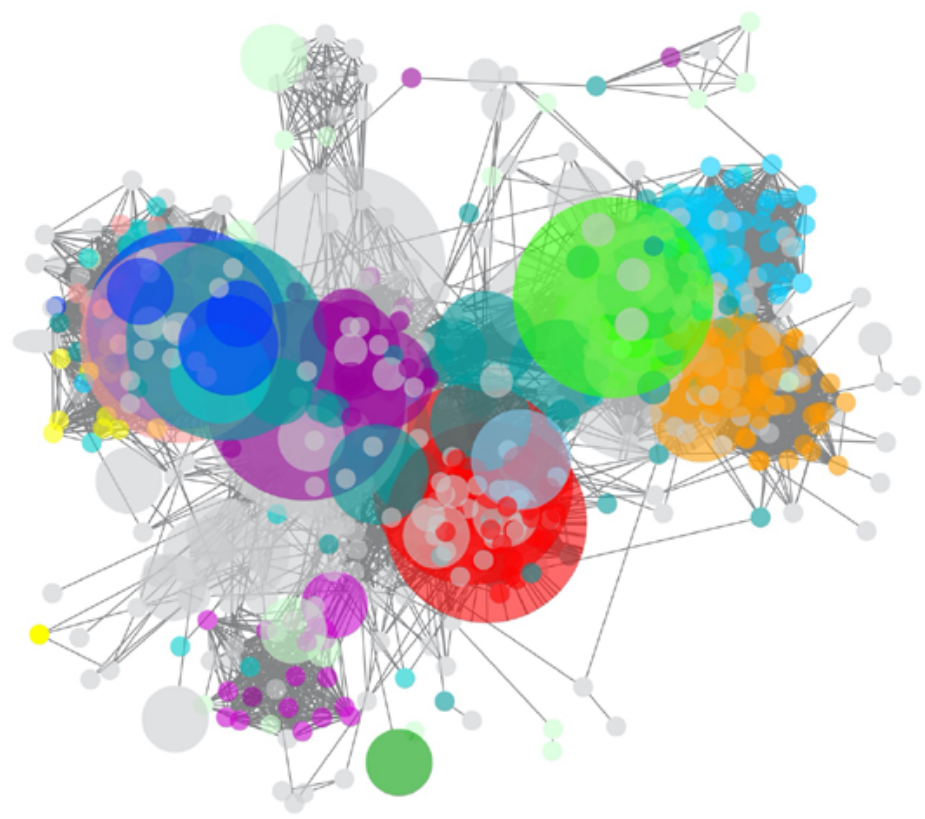

Figura 2. Estructura de comunidades de la red RON para las elecciones del 10 de Noviembre (10N) de 2019, con valor umbral de 0,01 (Vox-verde claro, PP-azul claro, Csnaranja, PSOE-rojo, UP-violeta, JuntsxCAT-turquesa, ERC-amarillo)

Fuente: Elaboración propia. 


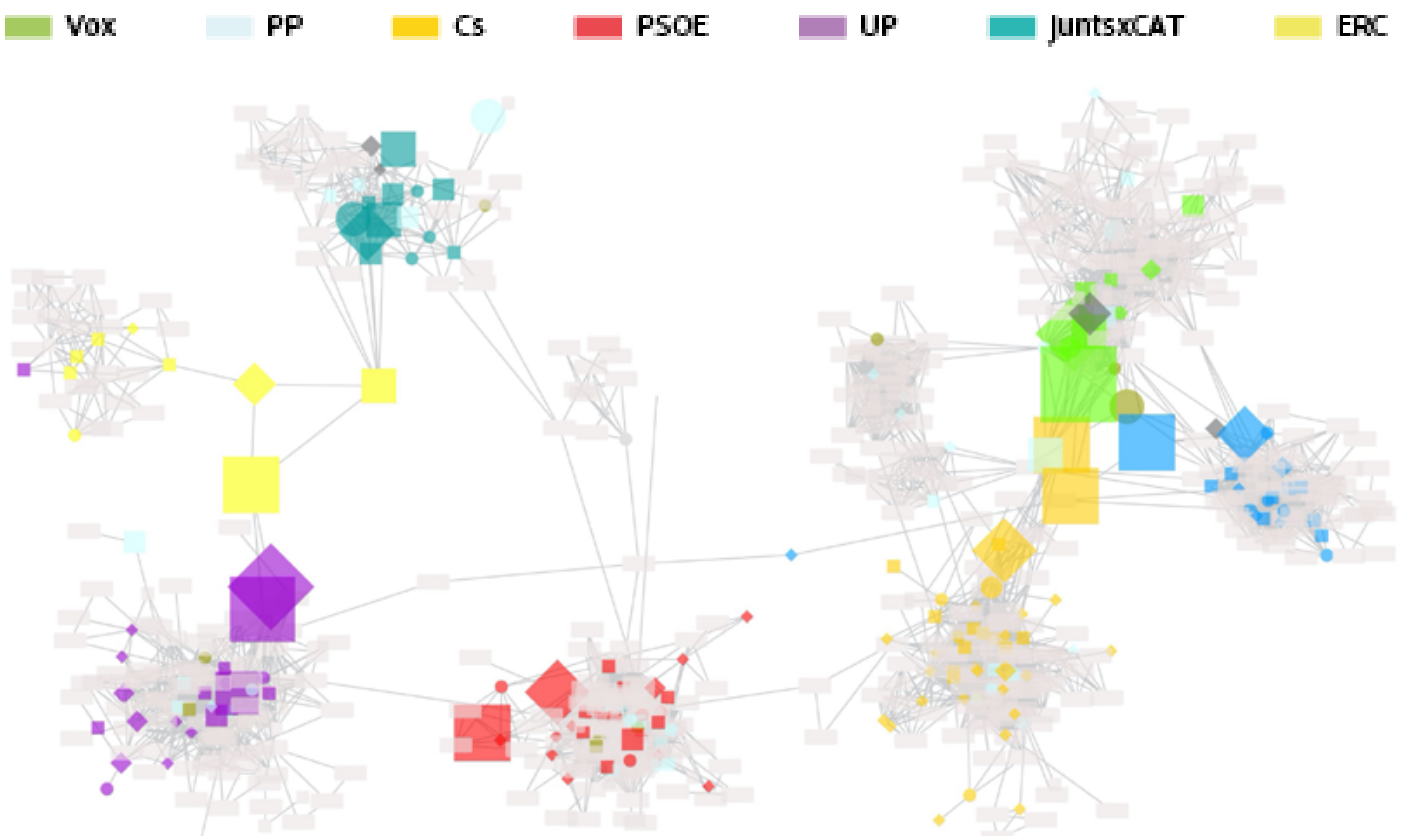

Figura 3. Estructura de comunidades de la red RON para las elecciones del 28 de Abril (28A) de 2019, con valor umbral de 0,05 (Vox-verde claro, PP-azul claro, Cs-naraja, PSOE-rojo, UP-violeta, JuntsxCAT-turquesa, ERC-amarillo)

Fuente: Elaboración propia.
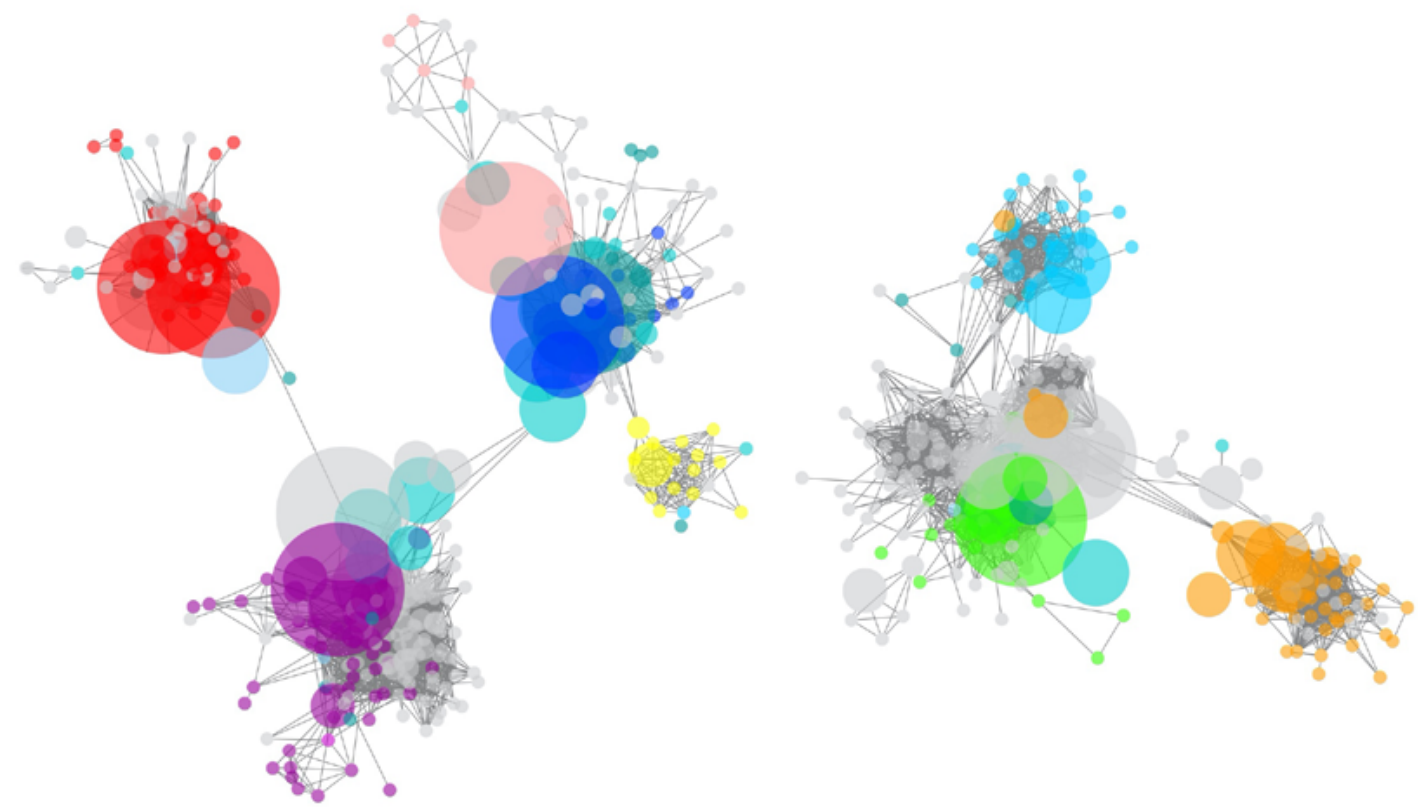

Figura 4. Estructura de comunidades de la red RON para las elecciones del 10 de Noviembre (10N) de 2019, con valor umbral de 0,05 (Vox-verde claro, PP-azul claro, Csnaraja, PSOE-rojo, UP-violeta, JuntsxCAT-turquesa, ERC-amarillo)

Fuente: Elaboración propia. 
Las diferencias entre las redes de 2019 y las de 2015 y 2016 son evidentes. En las figuras 5 y 6 podemos ver las estructuras de 2015 y 2016, cuando Vox tenía una presencia prácticamente nula y el partido de izquierda Podemos era una formación incipiente con una representación de 0 diputados en 2015 y de 42 en 2016. La comparación entre las figuras 3 y 4 , por un lado, y las figuras 5 y 6 , por otro, muestra cómo la irrupción de Vox (en verde) cambia sustancialmente las estructuras de debate de los partidos, que pasan de una estructura de polarización múltiple (figuras 5 y 6) a una estructura más polarizada en dos bloques (figuras 3 y 4).

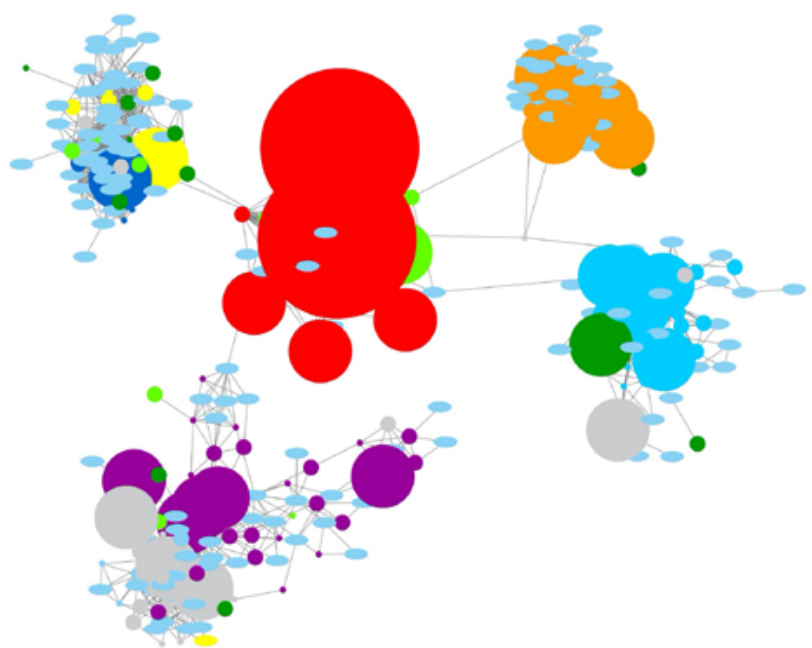

Figura 5. Estructura de comunidades de la red RON para las elecciones del 20 de diciembre (20D) de 2015, con valor umbral de 0,05

Fuente: Elaboración propia.

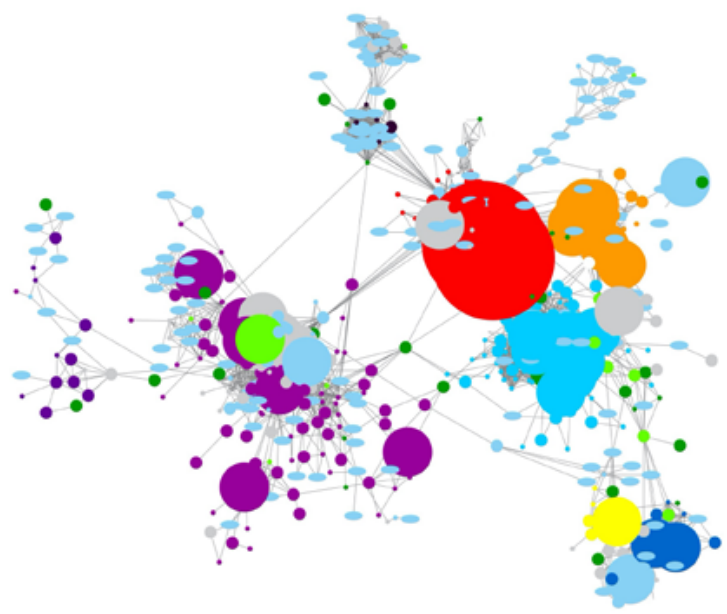

Figura 6. Estructura de comunidades de la red RON para las elecciones del 26 de Junio (26J) de 2016, con valor umbral de 0,05

Fuente: Elaboración propia. 
Para responder a la segunda pregunta de investigación, sobre las distancias ideológicas entre partidos en las elecciones de abril y noviembre de 2019 y las diferencias observadas entre estas distancias y las de las elecciones de 2015 y 2016, calculamos los valores de las intersecciones entre los principales partidos, agrupando las comunidades de retuiteadores de los miembros de cada partido. Esto permite aproximarse mejor a la distancia entre partidos, si asumimos que las intersecciones entre comunidades pueden ser un buen indicador de estas distancias (Guerrero-Solé \& López-González, 2019). En las tablas 2 y 3 se muestran las intersecciones entre las comunidades de retuiteadores de los partidos para las elecciones del 28 de abril y de 10 de noviembre de 2019, respectivamente.

\begin{tabular}{ccccccc} 
Partidos 28A & $\mathbf{1}$ & $\mathbf{2}$ & $\mathbf{3}$ & $\mathbf{4}$ & $\mathbf{5}$ & $\mathbf{6}$ \\
\hline $\operatorname{UPP}(1)$ & & & & & \\
\hline $\operatorname{ERC}(2)$ &, 0079 & & & & & \\
\hline JuntsxCAT (3) &, 0035 &, 0409 & & & & \\
\hline PSOE (4) &, 0127 &, 0011 & 0 & 0026 & & \\
\hline Cs (5) &, 0013 &, 0002 &, 0003 &, 0031 &, 0154 & \\
\hline $\operatorname{PP}(6)$ &, 0013 &, 0002 & 0 & 0013 &, 0203 &, 0268 \\
\hline $\operatorname{VOX}(7)$ &, 0006 & 0 & 0 & & \\
\hline
\end{tabular}

Tabla 2. Distancias entre los principales partidos políticos en abril de 2019

Fuente: Elaboración propia.

\begin{tabular}{|c|c|c|c|c|c|c|}
\hline Partidos $10 \mathrm{~N}$ & 1 & 2 & 3 & 4 & 5 & 6 \\
\hline \multicolumn{7}{|l|}{ UP (1) } \\
\hline $\operatorname{ERC}(2)$ & ,0034 & & & & & \\
\hline JuntsxCAT (3) & ,0036 & ,0157 & & & & \\
\hline PSOE (4) & ,0032 & ,0006 & ,0004 & & & \\
\hline $\operatorname{Cs}(5)$ & ,001 & ,0006 & ,0002 & ,0024 & & \\
\hline$P P(6)$ & ,0005 & ,0011 & 0 & ,0023 & ,01 & \\
\hline $\operatorname{vox}(7)$ & ,0004 & 0 & 0 & ,0010 & ,0058 & ,0125 \\
\hline
\end{tabular}

Tabla 3. Distancias entre los principales partidos políticos en noviembre de 2019

Fuente: Elaboración propia.

En las tablas 4 y 5 se muestran las intersecciones entre las comunidades de retuiteadores de los partidos para las elecciones de 2015 y 2016, respectivamente. 


\begin{tabular}{cccc} 
Partidos 10N & $\mathbf{1}$ & $\mathbf{2}$ & $\mathbf{3}$ \\
\hline UP (1) & & & \\
\hline PSOE (2) &, 0261 & & \\
\hline Cs (3) &, 0035 &, 0043 &, 0105 \\
\hline PP (4) &, 0028 &, 0034 & \\
\hline
\end{tabular}

Tabla 4. Distancias entre los principales partidos políticos en diciembre de 2015

Fuente: Elaboración propia.

\begin{tabular}{cccc} 
Partidos 10N & $\mathbf{1}$ & $\mathbf{2}$ & $\mathbf{3}$ \\
\hline UP (1) & & & \\
PSOE (2) &, 0066 & & \\
\hline $\operatorname{Cs}(3)$ &, 0023 &, 0134 &, 0358 \\
\hline PP (4) &, 0024 &, 0052 & \\
\hline
\end{tabular}

Tabla 5. Distancias entre los principales partidos políticos en junio de 2016

Fuente: Elaboración propia.

Como se puede ver en las diferentes tablas, el cálculo de las distancias ideológicas entre partidos confirma las estructuras visualizadas. La irrupción de Vox afecta principalmente a las distancias entre PSOE y Cs, pues pasa de una ratio de ,0134 en junio de 2016 (tabla 5) a ,0026 y ,0024 en las dos elecciones de 2019 (tablas 2 y 3). Esta disminución de la interacción se produce también entre el PSOE y el $\mathrm{PP}$, aunque no tan acentuada. Finalmente, estos datos muestran la configuración de dos grandes bloques en 2019 con una nula interacción entre ellos: el formado por la derecha española (PP, Vox y Cs) y el formado por la izquierda española (PSOE y Podemos) junto con los partidos nacionalistas ERC y JuntsxCAT, sin espacio para el centro.

\section{CONCLUSIONES}

Como se ha comprobado en estudios anteriores, los usuarios con una misma ideología política se estructuran en comunidades sensiblemente aisladas que evidencian un comportamiento altamente homofílico (Hanna et al., 2013; Bessi et al., 2016). Sin embargo, este estudio añade elementos de gran interés para la investigación en comunicación política en redes sociales: la emergencia de un partido populista de extrema derecha y la influencia que esta emergencia ha tenido en las estructuras de comunidades y en el movimiento de las distancias entre los diferentes partidos políticos, específicamente los de la derecha española (PP y Cs). 
Los resultados obtenidos permiten dar respuesta a las preguntas de investigación planteadas. En primer lugar, observamos cómo las redes de intersección de retuiteadores (RON) tienen una estructura de multitudes polarizadas, según la clasificación de Smith y sus colegas (2014). En las figuras 3 y 4 observamos la estructuración en clústeres de partidos políticos, para un valor umbral de 0,05, así como una clara polarización de esta red entre los partidos de centro-derecha, derecha y ultraderecha (Cs, PP, y VOX), y los partidos de centro-izquierda, izquierda y nacionalistas (PSOE, UP, ERC, JXCAT). Esta polarización es más acusada en las elecciones de noviembre de 2019 que en las de abril, en las que aún pueden observarse enlaces entre los dos grandes clústeres ideológicos. En cuanto a las diferencias con las elecciones de 2015 y 2016, observamos un distanciamiento entre los dos partidos que son percibidos, a priori, más próximos al centro político (CIS, 2019).

Comparando las redes RON de 2015 y 2016, sin la presencia de Vox, con las de 2019, observamos que el partido populista de ultraderecha ocupa un lugar central en el clúster de partidos de derecha, situándose cerca del PP y de Cs, y estos, a su vez, más alejados del PSOE, el partido de centro-izquierda. Así pues, podemos concluir que, al menos en lo referente a las redes de intersección de retuiteadores, la irrupción de Vox altera de manera sensible la estructura en comunidades y acerca la estructura en comunidades de la red al modelo de cámara de resonancia (Sunstein, 2017; Pariser, 2011; del Vicario et al., 2016).

En lo referente a las distancias entre los partidos políticos, que pueden servir de aproximación a la distancia ideológica entre partidos (Guerrero-Solé \& LópezGonzález, 2019), así como a la indecisión en el voto (Guerrero-Solé et al., 2014), podemos comprobar en las tablas 2 y 3 que los dos partidos más próximos entre sí en las elecciones de abril de 2019 fueron los partidos independentistas catalanes, ERC y JxCAT, seguidos por los partidos de la derecha política (Cs, PP y Vox). Aunque con intensidades diferentes, el patrón se repite en las elecciones de noviembre de 2019, con muy pocas variaciones. Del mismo modo, observamos una intersección nula entre los partidos independentistas catalanes y el populismo de ultraderecha de Vox, hecho que confirma que ningún usuario de Twitter que haya retuiteado a alguna de las cuentas independentistas analizadas ha retuiteado a su vez a alguna de las cuentas analizadas de Vox. Los datos de 2019 son significativamente diferentes de los de 2015 y 2016, en los que el PSOE aparecía como un partido próximo a UP, en 2015, y a Cs, en 2016. En conclusión, Vox parece haber cohesionado el clúster o familia política de la derecha española y, a su vez, ha debilitado los enlaces entre los partidos de izquierda y derecha, aumentando consecuentemente la polarización política medida en términos de intersección de comunidades de retuiteadores. Este aumento de la polarización va acompañado, además, de un discurso con cada vez mayores elementos de toxicidad (Guerrero-Solé \& Philippe, 2020; Castro Martínez \& Días Morilla, 2021) 
Atendiendo a los datos obtenidos en Twitter podemos concluir lo siguiente: (1) la política española de 2019 es una política más polarizada, dividida en dos grandes grupos ideológicos; (2) se han debilitado los enlaces entre partidos que se consideran próximos al centro; (3) el populismo de ultraderecha de Vox ocupa un lugar central en el clúster político de la derecha, y confirma su capacidad de adaptación a la lógica de los medios alternativos (Engesser et al., 2017b) y las redes sociales (Guerrero-Solé et al., 2020; Klinger \& Svensson, 2015). Entre las posibles consecuencias de la estructura y las distancias observadas en las redes, destacamos la adopción de estrategias de ultraderecha populista por parte de los partidos de derecha y centroderecha, así como la gran facilidad con la que se difunden los mensajes populistas en el clúster de derechas en España.

Evidentemente, esta investigación no está libre de limitaciones y sesgos derivados de la utilización de datos de Twitter, una red social cuya audiencia no puede ser considerada como una representación fidedigna de la población votante del estado español (para más detalles acerca de la naturaleza de las audiencias de las redes sociales en España, ver Moreno, 2021). Sin embargo, los resultados dan una buena aproximación en relación con la organización de los procesos de difusión de información a través de redes sociales, así como a las distancias y a las dinámicas de variación de las distancias entre partidos políticos. No hay duda de que muestras mayores y más representativas pueden ofrecer detalles más precisos sobre el proceso de circulación de información y sobre las conexiones entre políticos, medios y ciudadanía. Por ello, es importante también seguir con las reivindicaciones de un mayor acceso a los datos de todas las redes sociales para que la comunidad académica pueda analizar qué es lo que está ocurriendo en ellas sin necesidad de verse limitada por accesos insuficientes (Bruns, 2019a), como es el caso de Twitter, o por una falta absoluta de acceso como es el caso de Facebook o Instagram. Una última limitación es la ausencia de otros partidos con menor representación en el Congreso de los Diputados, pero que pueden ser clave para la gobernabilidad de España, como Más País, los partidos nacionalistas vasco y gallego, PNV y BNG, o la izquierda radical vasca, EH Bildu. En futuras investigaciones estas formaciones deberían ser tomadas en consideración.

\section{FINANCIAMIENTO}

Esta investigación ha sido financiada por el proyecto Polarización, duplicación de audiencias activas y populismo en Twitter. Análisis de la influencia de los discursos populistas en los debates políticos en España (2016-2020) (Datapop). PGC2018-097352-A-IO0 (MCIU / AEI / FEDER, UE- 2019-2021). 


\section{REFERENCIAS}

Abuín-Vences, N. \& García-Rosales, D. F. (2020). Elecciones generales de 2019 en Twitter: eficacia de las estrategias comunicativas y debates televisados como motor del discurso social (2019 general elections on Twitter: effectiveness of communicative strategies and televised debates as an engine of social discourse). El profesional de la información, 29(2), e290213. https://doi.org/10.3145/epi.2020.mar.13

Allcott, H. \& Gentzkow, M. (2017). Social Media and Fake News in the 2016 Election. Journal of Economic Perspectives, 31(2), 211-236. https://doi.org/10.1257/jep.31.2.211

Arcila Calderón, C., Van Atteveldt, W., \& Trilling, D. (2021). Dossier Métodos Computacionales y Big Data en la Investigación en Comunicación (Special Issue Computational Methods and Big Data in Communication Research). Cuadernos.info, (49), I-IV. https://doi.org/10.7764/cdi.49.35333

Atton, C. (2006). Far-right media on the Internet: culture, discourse and power. New Media E Society, 8(4), 573-587. https://doi.org/10.1177/1461444806065653

Berger, J. M. (2018). The alt-right Twitter census. Defining and Describing the Audience for AltRight Content on Twitter. A Vox Pol Report. https://www.voxpol.eu/download/vox-pol_ publication/AltRightTwitterCensus.pdf

Azaouzi, M., Rhouma, D., \& Ben Romdhane, L. (2019). Community detection in large-scale social networks: state-of-the-art and future directions. Social Network Analysis and Mining, 9(23). https://doi.org/10.1007/s13278-019-0566-X

Bakshy, E., Messing, S., \& Adamic, L. (2015). Exposure to ideologically diverse news and opinion on Facebook. Science, 348(6239), 1130-1132. https://doi.org/10.1126/science.aaal160

Barberá, P., Jost, J. T., Nagler, J., Tucker, J. A., \& Bonneau, R. (2015). Tweeting From Left to Right: Is Online Political Communication More Than an Echo Chamber? Psychological Science, 26(10), 1531-1542. https://doi.org/10.1177/0956797615594620

Bessi, A., Zollo, F., Del Vicario, M., Puliga, M., Scala, A., Caldarelli, G., Uzzi, B., \& Quattrociocchi, W. (2016). Users polarization on Facebook and YouTube. PLoS ONE, 11(8), e0159641. https://doi.org/10.1371/journal.pone.0159641

Boutet, A., Kim, H., \& Yoneki, E. (2012). What's in Twitter: I Know What Parties are Popular and Who You Are Supporting Now! In 2012 IEEE/ACM International Conference on Advances in Social Networks Analysis and Mining (pp. 132-139). https://doi.org/10.1109/ASONAM.2012.32

Boxell, L., Gentzkow, M., \& Shapiro, J. M. (2017). Greater Internet use is not associated with faster growth in political polarization among US demographic groups. PNAS, 114(40), 10612-10617. https://doi.org/10.1073\%2Fpnas.1706588114

Bracciale, R. \& Martella, A. (2017). Define the populist political communication style: the case of Italian political leaders on Twitter. Information Communication and Society, 20 (9), 1310-1329. https://doi.org/10.1080/1369118X.2017.1328522

Bruns, A. (2019a). After the 'APIcalypse': social media platforms and their fight against critical scholarly research. Information Communication and Society, 22(11), 1544-1566. https://doi.org/10.1080/1369118X.2019.1637447 
Bruns, A. (2019b). Are Filter Bubbles Real? (Digital Futures Series). Polity.

Caiani, M. \& Kröll, P. (2015). The transnationalization of the extreme right and the use of the Internet. International. Journal of Comparative and Applied Criminal Justice, 39(4), 331-351. https://doi.org/10.1080/01924036.2014.973050

Caiani, M. \& Wagemann, C. (2009). Online networks of the Italian and German extreme right. Information Communication \& Society, 12(1), 66-109. https://doi.org/10.1080/13691180802158482

Caiani, M. \& Parenti, L. (2013). European and American Extreme Right Groups and the Internet. Ashgate Publishing Limited.

Castro Martínez, A. \& Días Morilla, P. (2021). La comunicación política de la derecha radical en redes sociales. De Instagram a TikTok y Gab, la estrategia digital de Vox (The political communication of the right-wing radicals on social networks. From Instagram to TikTok and Gab, Vox's digital strategy). Dígitos. Revista de Comunicación Digital, (7), 67-89. https://doi.org/10.7203/rd.v1i7.210

CIS. (2019). Barómetrodenoviembre 2019. Estudion³267 (Barometer of November 2019. Study n³267). http://www.cis.es/cis/export/sites/default/-Archivos/Marginales/3260_3279/3267/es3267mar.pdf

Conover, M., Ratkiewicz, J., Francisco, M., Gonçalves, B., Flammini, A., \& Menczer, F. (2011). Political polarization on Twitter. Proceedings of the International AAAI Conference on Webl and Social Media, 5(1), 89-96. https://ojs.aaai.org/index.php/ICWSM/article/view/14126

Crosset, V., Tanner, S., \& Campana, A. (2019). Researching far right groups on Twitter: Methodological challenges 2.0. New Media \& Society, 21(4), 939-961. https://doi.org/10.1177/1461444818817306

Daniels, J. (2018). The algorithmic rise of the 'alt-right'. Contexts, 17(1), 60-65. https://doi.org/10.1177/1536504218766547

Del Vicario, M., Bessi, A., Zollo, F., Petroni, F., Scala, A., Caldarelli, G., Stanley, H. E., \& Quattrociocchi, W. (2016). The spreading of misinformation online. PNAS, 113(3), 554-559. https://doi.org/10.1073/pnas.1517441113

Engesser, S., Ernst, N., Esser, F., \& Büchel, F. (2017a). Populism and social media: how politicians spread a fragmented ideology. Information, Communication \& Society, 20(8), 1109-1126. https://doi.org/10.1080/1369118X.2016.1207697

Engesser, S., Ernst, N., Esser, F., \& Büchel, F. (2017b). Extreme parties and populism: an analysis of Facebook and Twitter across six countries. Information, Communication $\mathcal{E}$ Society, 20(9), 1347-1364. https://doi.org/10.1080/1369118X.2017.1329333

Faris, R. M., Roberts, H., Etling, B., Bourassa, N., Zuckerman, E., \& Benkler, Y. (2017). Partisanship, propaganda, and disinformation: Online Media and the 2016 U.S. Presidential Election. Berkman Klein Center for Internet. http://nrs.harvard.edu/urn-3:HUL.InstRepos:33759251

Ferreira, C. (2019). Vox como representante de la derecha radical en España: un estudio sobre su ideología (Vox as a representative of the radical right in Spain: a study of its ideology). Revista Española de Ciencia Política, (51), 73-98. https://doi.org/10.21308/recp.51.03

Fortunato, S. (2010). Community detection in graphs. Physics Reports, 486(3-5), 75-174. https://doi.org/10.1016/j.physrep.2009.11.002 
Froio, C. \& Ganesh, B. (2019). The transnationalisation of far right discourse on Twitter. Issues and actors that cross borders in Western European democracies. European Societies, 21(4), 513-539. https://doi.org/10.1080/14616696.2018.1494295

Golder, M. (2016). Far Right Parties in Europe. Annual Review of Political Science, 19(1), 477-497. https://doi.org/10.1146/annurev-polisci-042814-012441

Gómez-Domínguez, P., Besalú-Casademont, R., \& Guerrero-Solé, F. (2016). Social metamedia and public service: The debate in Twitter for television programs on the BBC, RTVE, and CCMA. El profesional de la información, 25(3), 384-394. https://doi.org/10.3145/epi.2016.may.08

Guerrero-Solé, F. (2017). Community detection in political discussions on Twitter. An application of the Retweet Overlap Network method to the Catalan process towards independence. Social Science Computer Review, 35(2), 244-261. https://doi.org/10.1177/0894439315617254

Guerrero-Solé, F. (2018). Interactive Behavior in Political Discussions on Twitter: Politicians, Media, and Citizens' Patterns of Interaction in the 2015 and 2016 Electoral Campaigns in Spain. Social Media + Society, 4(4). https://doi.org/10.1177/2056305118808776

Guerrero-Solé, F., Corominas-Murtra, B., \& Lopez-Gonzalez, H. (2014). Pacts with Twitter. Predicting voters' indecision and preferences for coalitions in multiparty systems. Information, Communication \& Society, 17(10), 1280-1297. https://doi.org/10.1080/1369118X.2014.920040

Guerrero-Solé, F. \& López-González, H. (2019). Government Formation and Political Discussions in Twitter. An Extended Model for Quantifying Political Distances in Multiparty Democracies. Social Science Computer Review, 37(1), 3-21. https://doi.org/10.1177/0894439317744163

Guerrero-Solé, F. \& Philippe, O. (2020). La toxicidad de la política española en Twitter durante la pandemia de la COVID-19. Hipertext.net, 21, 133-139. https://doi.org/10.31009/hipertext.net.2020.i21.12

Guerrero-Solé, F., Suárez-Gonzalo, S., Rovira, C., \& Codina, L. (2020). Social media, context collapse and the future of data-driven populism. Profesional de la información, 29(5), e290506. https://doi.org/10.3145/epi.2020.sep.06

Hanna, A., Wells, C., Maurer, P., Friedland, L., Shah, D., \& Matthes, J. (2013). Partisan alignments and political polarization online: A computational approach to understanding the french and US presidential elections. In PLEAD '13: Proceedings of the 2nd workshop on Politics, elections and data (pp. 15-22). https://doi.org/10.1145/2508436.2508438

Hedgecoe, G. (2019, November 11). Spanish elections: How the far-right Vox party found its footing. BBC. https://www.bbc.com/news/world-europe-46422036

Ikenberry, G. J. (2018). The end of liberal international order? International Affairs, 94(1), 7-23. https://doi.org/10.1093/ia/iix241

Jones, S. (2019, April 14). Far-right Vox party set for breakthrough in Spanish elections. The Guardian. https://www.theguardian.com/world/2019/apr/14/spain-vox-party-oncourse-to-break-into-mainstream-politics

Jungherr, A. (2014). Twitter in politics: A comprehensive literature review. SSRN, 2865150. https://doi.org/10.2139/ssrn.2402443 
Klinger, U. \& Svensson, J. (2015). The emergence of network media logic in political communication: A theoretical approach. New Media and Society, 17(8), 1241-1257. https://doi.org/10.1177\%2F1461444814522952

Marín-Dueñas, P. P., Simancas-González, E., \& Berzosa-Moreno, A. (2019). Uso e influencia de Twitter en la comunicación política: el caso del Partido Popular y Podemos en las elecciones generales de 2016 (Twitter and political communication: the case of the Partido Popular and Podemos in the 2016 general elections). Cuadernos.info, (45), 129-144. https://doi.org/10.7764/cdi.45.159

Mas Manchón, L. \& Guerrero-Solé, F. (2019). The use of hashtags as a political branding strategy. Revista Internacional de Relaciones Públicas, 9(17), 5-24. https://doi.org/10.5783/RIRP-17-2019-02-05-24

McSwiney, J. (2020). Social networks and digital organisation: far right parties at the 2019 Australian federal election. Information, Communication \& Society, 24(10), 1401-1418. https://doi.org/10.1080/1369118X.2020.1757132

Minder, R. (2019, November 10). Spain's Far Right Gains in Election. New York Times. https://www.nytimes.com/2019/11/10/world/europe/spain-election.html

Moreno, E. (2021). Los servicios de mensajería instantánea se consolidan para leer, ver, encontrar, compartir o comentar noticias. https://www.digitalnewsreport.es/2021/ los-servicios-de-mensajeria-instantanea-se-consolidan-para-leer-ver-encontrarcompartir-o-comentar-noticias/

Mudde, C. (2004). The Populist Zeitgeist. Government and Opposition. An International Journal of Comparative Politics, 39(4), 542-563. https://doi.org/10.1111/j.1477-7053.2004.00135.x

Norris, P. (2005). Radical Right: Voters and Parties in the Electoral Market. Cambridge University Press. https://doi.org/10.1017/CBO9780511615955

Pariser, E. (2011). The Filter Bubble. What the Internet Is Hiding from You. Penguin.

Pérez Curiel, C. (2020). Trend towards extreme right-wing populism on Twitter. An analysis of the influence on leaders, media and users. Communication \& Society, 33(2), 175-192. https://doi.org/10.15581/003.33.2.175-192

Pierri, F., Artoni, A., \& Ceri, S. (2020). Investigating Italian disinformation spreading on Twitter in the context of 2019 European elections. PLoS ONE 15(1), e0227821. https://doi.org/10.1371/journal.pone.0227821

Raghavan, U. N., Albert, R., \& Kumara, S. (2007). Near linear time algorithm to detect community structures in large-scale networks. Physical Review E - Statistical, Nonlinear, and Soft Matter Physics, 76(3), [036106]. https://doi.org/10.1103/PhysRevE.76.036106

Rinken, S. (2019). Actitudes ante la inmigración y comportamiento electoral en España (Attitudes towards immigration and electoral behavior in Spain). In Anuario CIDOB de la Inmigración (pp. 68-84). https://doi.org/10.24241/AnuarioCIDOBInmi.2019.68

Rivas-de-Roca, R., García-Gordillo, M., \& Bezunartea-Valencia, O. (2020). The far-right's influence on Twitter during the 2018 Andalusian elections: an approach through political leaders. Communication \& Society, 33(2), 227-242. https://doi.org/10.15581/003.33.2.227-242 
Rooduijn, M. (2014). The mesmerizing message: The diffusion of populism in public debates in western European media. Political Studies, 62(4), 726-744.

https://doi.org/10.1111\%2F1467-9248.12074

Smith, M. A., Rainie, L., Shneiderman, B., \& Himelboim, I. (2014). Mapping Twitter topic networks: From polarized crowds to community clusters. The Pew Research Center. https://www.pewresearch.org/internet/2014/02/20/mapping-twitter-topic-networksfrom-polarized-crowds-to-community-clusters/

Suiter, J., Culloty, E., Greene, D., \& Siapera, E. (2018). Hybrid media and populist currents in Ireland's 2016 General Election. European Journal of Communication, 33(4), 396-412. https://doi.org/10.1177/0267323118775297

Sunstein, C. R. (2001). Echo chambers: Bush v. Gore, impeachment, and beyond. Princeton University Press.

Sunstein, C. R. (2009). Republic.com. Princeton University Press.

Sunstein, C. R. (2017). \#Republic: Divided Democracy in the Age of Social Media. Princeton University Press.

Taggart, P. (2000). Populism. Open University Press.

Tucker, J., A., Theocharis, Y., Roberts, M. E., \& Barberá, P. (2017). From liberation to turmoil: social media and democracy. Journal of democracy, 28(4), 46-59. https://muse.jhu.edu/article/671987

Tumasjan, A., Sprenger, T. O., Sandner, P. G., \& Welpe, I. (2010). Predicting elections with Twitter: What 140 characters reveal about political sentiment. In Proceedings of the Fourth International AAAI Conference on Weblogs and Social Media (pp. 178-185).

https://www.aaai.org/ocs/index.php/ICWSM/ICWSM10/paper/viewFile/1441/1852

Turnbull-Dugarte, S. J. (2019). Explaining the end of Spanish exceptionalism and electoral support for Vox. Research and Politics, 6(2), 1-8. https://doi.org/10.1177/2053168019851680

Vampa, D. (2020). Competing forms of populism and territorial politics: the cases of Vox and Podemos in Spain. Journal of Contemporary European Studies, 28(3), 304-321. https://doi.org/10.1080/14782804.2020.1727866

von Beyme, K. (1988). Right-Wing Extremism in Post-War Europe. West European Politics, 11(2), 1-18. https://doi.org/10.1080/01402388808424678

Xie, J. \& Szymanski, B. K. (2011). Community detection using a neighborhood strength driven label propagation algorithm. In 2011 IEEE Network Science Workshop (pp. 188-197). https://doi.org/10.1109/NSW.2011.6004645

Wojcieszak, M. \& Mutz, D. C. (2009). Online groups and political discourse: Do online discussion spaces facilitate exposure to political disagreement? Journal of Communication, 59(1), 40-56. https://doi.org/10.1111/j.1460-2466.2008.01403.x 


\section{SOBRE LOS AUTORES}

FREDeRIC GUERRERO-SOLÉ, es doctor en Comunicación Publica por la Universidad Pompeu Fabra (UPF), y profesor de Sociología de la Comunicación y Medios Digitales y Relaciones Públicas en esta misma universidad. Sus principales líneas de investigación son redes sociales y comunicación política, y efectos de los medios. Ha publicado artículos en numerosas revistas científicas como Information, Communication \& Society, Social Science Computer Review y Social Media + Society. Actualmente es el subdirector de investigación del departamento de comunicación de la UPF.

\section{https://orcid.org/0000-0001-8145-8707}

LLUÍS MAS-MANCHóN ,es profesor en el Departamento de Comunicación de la Universidad Pompeu Fabra (Barcelona). Sus principales intereses de investigación son las estrategias de branding, la publicidad y la comunicación estratégica en medios digitales. Lluís Mas ha publicado artículos en revistas internacionales como Journal of Product and Brand Management, American Behavioral Scientist o Corporate Reputation Review. Obtuvo una beca para ser profesor visitante en la Texas Tech University (2017) y recientemente ha editado el libro Innovation in Advertising and Branding Communication (2020, Routledge).

Dttp://orcid.org/0000-0003-2239-4082

TONI AIRA, Periodista, doctor en comunicación por la Universitat Ramon Llull. Director y profesor del Máster en Comunicación Política e Institucional de la UPF Barcelona School of Management (UPFBSM) y Director de la Cátedra Agbar de estudios internacionales en comunicación institucional para el desarrollo y crecimiento sostenible, de la UPF-BSM. Profesor asociado en los Grados de Comunicación y de Ciencias Políticas y de la Administración en la Universitat Pompeu Fabra (UPF). Miembro del Grupo de Investigación en Comunicación Política, Periodismo y Democracia (POLCOM-GRP).

iD https://orcid.org/0000-0001-9199-9339 